\title{
Numerical solutions to Giovannini's QCD parton branching processes
}

Z. Ong ${ }^{1, *}, P$. Agarwal ${ }^{1}, H . W . \mathrm{Ang}^{1}, E . \mathrm{Wang}^{1}, A . H . \mathrm{Chan}^{1}$, and C.H. $\mathrm{Oh}^{1}$

${ }^{1}$ Department of Physics, National University of Singapore. 2 Science Drive 3, Singapore 117551.

\begin{abstract}
In 1979, Giovannini [1] formulated multiparticle production as a manifestation of four QCD processes: $A: g \rightarrow g g$ (gluon bremsstrahlung), $\widetilde{A}: q \rightarrow q g$ (quark bremsstrahlung), $B: g \rightarrow \bar{q} q$ (quark pair creation) and $C: g \rightarrow g g g$ (four-gluon vertex). At present, only approximate solutions exist, such as the Generalized Multiplicity Distribution (GMD) which excludes processes $B$ and $C[2,3]$. These approximate solutions have been very successful in describing charged particle multiplicities at lower energies, at least until the appearance of a shoulder-like structure at $\sqrt{s} \approx 200 \mathrm{GeV}$ and $900 \mathrm{GeV}$ first reported by the UA5 collaboration [4], also known as "KNO-scaling violation". Here, we extend the work of Sakai [5] and attempt a numerical solution that incorporates all four QCD processes, and see if the addition of processes $B$ and $C$ can explain the shoulder-like structure.
\end{abstract}

\section{Introduction}

Giovannini's formulation of multiparticle production (as described in the abstract) can be cast into a differential-difference equation. For $m$ quarks and $n$ gluons at QCD evolution parameter $t$, we have:

$$
\begin{aligned}
\frac{\partial P_{m, n}}{\partial t}= & -(A n+\widetilde{A} m+B n+C n) P_{m, n} \\
& +A(n-1) P_{m, n-1} \\
& +\widetilde{A} m P_{m, n-1} \\
& +B(n+1) P_{m-2, n+1} \\
& +C(n-2) P_{m, n-2}
\end{aligned}
$$

where $A, \widetilde{A}, B$ and $C$ are the probabilities of the processes taking place. To investigate how KNO-scaling violation might arise from these four fundamental processes, we will integrate eq. (1) numerically using the $4^{\text {th }}$-order Runge-Kutta method (RK4) and study how varying $A, \widetilde{A}, B$ and $C$ modifies the final distribution, observing for the process(es) that might be responsible for producing the shoulder-like structure.

\footnotetext{
*e-mail: ongzongjin@u.nus.edu
} 


\section{Procedure}

First, we set up a 2-dimensional probability distribution $P_{2 D}[m][n]$, where $m=$ number of quarks and $n=$ number of gluons. For an initial condition of 2 quarks and 0 gluons (as an example), we set $P_{2 D}[2][0]=1$ and all other $P_{2 D}[m][n]=0$. The entire array is then integrated element-wise using RK4 from $t=0.0$ to 3.0 (the upper limit is arbitrarily set), with step size $h=0.01$.

$P_{2 D}[m][n]$ is then condensed into a parton distribution $P_{\text {parton }}(n)$, where both quarks and gluons are categorised together. $P_{\text {parton }}(n)$ is truncated at $n=M X$ (identified as the "kinematical bound" in [5]), which is set at $M X=100$ in this work to reflect finite branching. The truncated $P_{\text {parton }}(n)$ is then normalised to 1 .

Next, we convert the parton distribution to an all-hadron distribution $P_{h}(n)$. Each parton hadronises into $k$ hadrons according to a Poisson distribution (with average value $\lambda=2.5$ and maximum value $k_{\max }=7$, following [5]):

$$
P(k)=\frac{\frac{\exp (-\lambda) \cdot \lambda^{k}}{k !}}{\sum_{k=1}^{7} \frac{\exp (-\lambda) \cdot \lambda^{k}}{k !}}
$$

The all-hadron distribution $P_{h}(n)$ is then converted into a charged-hadron distribution $P_{c}\left(n_{c}\right)$. As before, we adopt the model used in [5]: pairs of $(+,-)$ or $(0,0)$ occur in equal probability, and we assume that the initial state contains an even number of charged particles. This constraints the final state to contain an even number of charged particles due charge conservation. If the final state has $(2 n+1)$ hadrons, the final odd hadron must then be neutral. The charged-hadron distribution is then computed from the all-hadron distribution using combinatorics:

$$
P_{c}\left(2 n_{c}\right)=\sum_{n \geq n_{c}} \frac{n !}{n_{c} !\left(n-n_{c}\right) ! 2^{l}}\left[P_{h}(2 n)+P_{h}(2 n+1)\right]
$$

\section{Plots}

In each of the plots that follow, we vary a selected parameter against a base configuration: $A=0.2, \widetilde{A}=0.2, B=0, C=0, M=2$ and $N=0$. Each figure shows a selected parameter taking three different values (one low, one intermediate and one high, labelled within the plot), with the other parameters taking the default values from the base configuration.
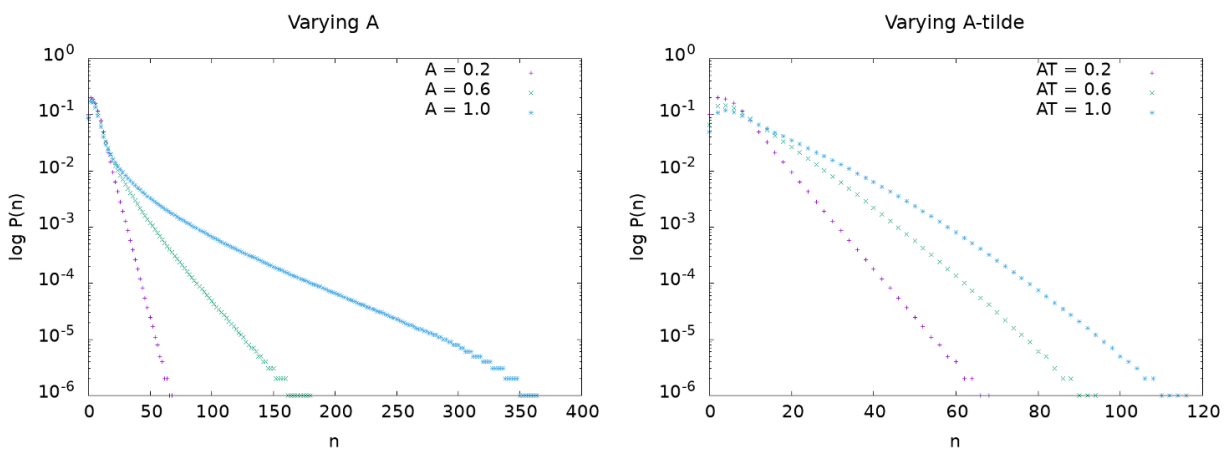

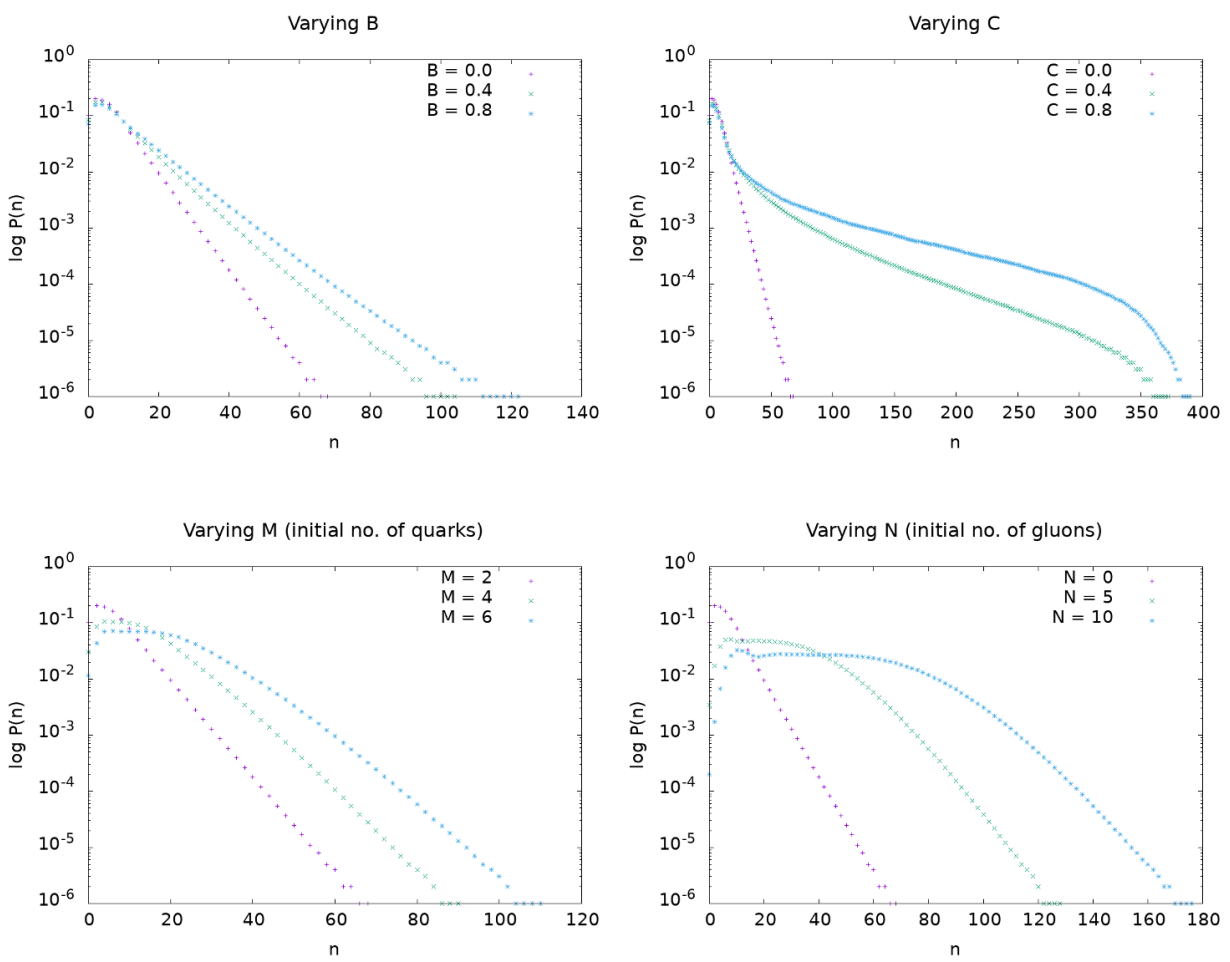

\section{Summary of Observations}

From the plots shown, we note the resulting effects of varying the six parameters:

1. Processes $A(g \rightarrow g g)$ and $C(g \rightarrow g g g)$ create the shoulder-like structure very strongly (a sharp change in gradient at approximately $n=25$ ). This suggests that at onset of $\mathrm{KNO}$-scaling violation, gluonic activity increases.

2. Processes $\widetilde{A}(q \rightarrow q g)$ and $B(g \rightarrow \bar{q} q)$ generally broaden the entire distribution.

3. Higher values of $M$ and $N$ have the effect of shifting peak multiplicity to the right, while maintaining an overall bell shape of multiplicity distribution.

\section{Conclusions}

The emergence of gluonic activity (processes $A$ and $C$ ) at the onset of KNO-scaling violation agrees with earlier predictions by Chan \& Chew [2]. They observed that as $\sqrt{s}$ increased towards the $\mathrm{TeV}$ range, fits of collider data to the GMD revealed decreasing $k$ and increasing $k^{\prime}$ (i.e. as gluon activity increases, distribution becomes more FYD).

In addition, we now have a method to observe theoretical distributions incorporating all four QCD processes, which also has the ability to reproduce the shoulder-like structure in KNO-scaling violation. This presents an alternate approach to the multicomponentNBD/FYD/GMD models used to describe the shoulder-like structure. 


\section{Future Work}

This preliminary work has hinted at a mechanism that possibly explains the onset of KNOscaling violation, which warrants further investigation. We outline two areas of further development.

Fits to collider data: we can use the method outlined to perform best-fits for $A, \widetilde{A}, B$, $C, M$ and $N$ against multiplicity plots from collider data. This potentially reveals whether gluonic activity indeed increases with collision energy. We can also do the same for different types of collisions (e.g. $p \bar{p}, e^{+} e^{-}, p A, A A$ ).

Reduced phase space: So far, our model produces even-multiplicity plots, which correspond to full phase-space multiplicities. The model can be modified to produce multiplicity plots for all- $n$, which is more common today as colliders report charged particle multipliticies from reduced $\eta$-space.

\section{Acknowledgements}

The authors would like to thank the National University of Singapore, and the support and helpful discussions with colleagues. This work is supported by the NUS Research Scholarship.

\section{References}

[1] A. Giovannini, Nucl. Physics, Sect. B 161, 429 (1979)

[2] A.H. Chan, C.K. Chew, Phys. Rev. D (1990)

[3] A. Dewanto, A.H. Chan, C.H. Oh, R. Chen, K. Sitaram, Eur. Phys. J. C 57, 515 (2008)

[4] UA5 collaboration, Phys. Lett. B (1986)

[5] S. Sakai, Phys. Rev. D (1989) 